\title{
Clinical associations of serum leptin and leptin/adiponectin ratio in systemic sclerosis
}

\author{
Małgorzata Michalska-Jakubus ${ }^{1}$, Karolina Sawicka ${ }^{1}$, Emilia Potembska², Małgorzata Kowal' ${ }^{1}$ Dorota Krasowska ${ }^{1}$
}

'Department of Dermatology, Venereology and Pediatric Dermatology, Medical University of Lublin, Lublin, Poland

2Department of Psychiatric Nursing, Medical University of Lublin, Lublin, Poland

Adv Dermatol Allergol 2019; XXXVI (3): 325-338

DOI: https://doi.org/10.5114/ada.2018.75809

\begin{abstract}
Introduction: Leptin and adiponectin have recently received the attention of researchers as attractive biomarkers in systemic sclerosis (SSc) because of their role in the inflammatory process, vascular function and fibrosis. We hypothesized that leptin and adiponectin may be associated with disease activity and severity in patients with SSc. Aim: To compare serum leptin, adiponectin and leptin/adiponectin levels in patients with SSc and healthy controls and to evaluate their possible relationship with frequently used laboratory markers and clinical findings.

Material and methods: The study included 48 Caucasian female patients with SSc and 38 healthy controls. Serum concentrations of leptin and adiponectin were measured in patients and controls using commercially available ELISA Kits (Quantikine ELISA Kit R\&D Systems, Minneapolis, MN, USA). The results were assessed by the Mann-Whitney $U$-test and Spearman's correlation test.

Results: Leptin and adiponectin levels correlated with body mas index in SSc patients $(r=0.495, p=0.000398$ and $r=-0.306 ; p=0.0342)$ in contrast to healthy controls ( $p=0.070$ and $p=0.256$, respectively), and, in SSc patients only, a strong negative correlation was observed between leptin and adiponectin serum levels $(r=-0.314$; $p=0.0312$ ). Diffuse form of the disease (dcSSc) was associated with significantly lower serum adiponectin levels (8638.62 \pm 10382.62 ). Active disease was associated with significantly lower leptin concentration (13700.49 $\pm 18293.32)$ and there was a significant negative correlation between leptin serum level and activity index score $(r=-0.342 ; p=0.0185)$.

Conclusions: The results of our study indicate that leptin levels might correlate with disease activity and subtype in SSc patients.
\end{abstract}

Key words: leptin, adiponectin, systemic sclerosis, disease activity.

\section{Introduction}

Systemic sclerosis (SSc) is a multiorgan connective tissue disease characterized by vascular injury and chronic autoimmune inflammation followed by excessive extracellular matrix deposition and ultimately progressive fibrosis of skin and internal organs. The etiology of SSc is still obscure, although genetic and environmental factors seem to underlay its pathogenesis [1]. In recent years, mediators synthesized in the adipose tissue, called adipocytokines, have arisen as new players in modulating immune responses and have been reported to play important roles in the pathogenesis of autoimmune rheumatic diseases [2-7].
Adipocytokines (or adipokines) are biologically active hormones secreted by adipocytes. Notably, two members of this family, namely leptin and adiponectin, seem to be attractive, since they are found to have opposing action. It is generally accepted that in most inflammatory diseases leptin displays an enhancing effect in inflammatory and immune responses, while adiponectin is considered to act primarily as an anti-inflammatory molecule $[2,3,6,8,9]$.

Leptin, a $16-\mathrm{kD}$ non-glycosylated polypeptide, is a cytokine-like hormone, synthesized mainly by the adipose tissue cells and primarily involved in regulating food intake, basal metabolism and body weight [3, 5, 10, 11]. Thus, the serum concentration of this hormone depends on the percentage of adipose tissue (it is positively cor-

Address for correspondence: Karolina Sawicka MD, Department of Dermatology, Venereology and Pediatric Dermatology, Medical University of Lublin, 13 Radziwitłowska St, 20-080 Lublin, Poland, phone/fax: +48 8153236 47, e-mail: k.sawicka10@gmail.com Received: 20.03.2018, accepted: 6.04.2018. 
related with body mass index (BMI)), but also on the patient's age and sex $[6,12]$. It structurally and functionally resembles the interleukin 6 (IL-6) cytokine family and binds to the receptor that is a member of the class I cytokine receptor family $[3,10-12]$. In the recent years, it has become apparent that the leptin receptor is widely expressed in all cell types of innate and adaptive immunity [13-16]. The interactions between leptin and inflammation are bidirectional. Leptin synthesis in humans shows a detectable increase as a response to acute inflammation, and secretion of inflammatory mediators such as IL-1, IL- 6 and tumor necrosis factor- $\alpha$ (TNF- $\alpha$ ). Leptin, in turn, up-regulates the production of pro-inflammatory cytokines such as IL-6, IL-12, and TNF- $\alpha$, thus perpetuating the loop of inflammation. In innate immunity, leptin also activates proliferation of monocytes, macrophages, chemotaxis of neutrophils, maturation of dendritic cells and cytotoxicity of natural killer cells (NK) [3, 5, 6, 10, $11,13,14,17,18]$. In adaptive immune responses, leptin may exert direct effects on lymphocytes. It stimulates secretion and maturation of thymocytes and T cell proliferation and polarizes ThO towards a Th1 response with production of pro-inflammatory cytokines such as interferon $\gamma($ INF- $\gamma)$ and IL-2 [13, 14, 19, 20]. Regarding B cells and immunoregulation, leptin activates them to produce cytokines such as IL-6, IL-10 and TNF- $\alpha$ [21]. Furthermore, leptin increases expression of adhesion molecules such as intercellular adhesion molecule-1 (ICAM-1) and very late antigen-2 (VLA2), which contributes to activation and migration of the cells to the inflammation site [13, 22]. As a result, Th1 and B cells' immune response stimulation may lead to the development and progression of autoimmune processes. Moreover, leptin is able to reduce the human CD4+CD25+ Treg cells, which are a small subset of CD4+ T cells that control the peripheral immune tolerance, and prevent inappropriate immune responses, such as allergy and autoimmunity $[13,14]$. In fact, experimental investigations on animal models have clearly indicated that leptin can promote autoreactivity [13]. In animals with adaptive immunity-mediated inflammation antigen-induced arthritis, or other autoimmune diseases, leptin deficiency has a protective effect by resulting in reduced production of proinflammatory Th1 cytokines and a shift toward a Th2 response [23]. Additionally, leptindeficient ob/ob mice and leptin receptor-deficient $\mathrm{db} / \mathrm{db}$ mice are resistant to the development of several experimentally induced autoimmune diseases [13].

Adiponectin is a biologically active protein built of 244 amino acids that form a trimer of $30 \mathrm{kDa}$ weight [6, $7,9]$. Its structure is similar to collagen VIII and X as well as the C1q complement component. It is widely produced by adipocytes, but also by muscles, cardiac myocytes and endothelial cells [4]. Adiponectin acts via three receptors: AdipoR1 (expressed most abundantly in skeletal muscles), AdipoR2 (expressed in the liver) and T-cadherin (mainly found in the heart and arteries) [24, 25]. Oppo- site to leptin, adiponectin acts as a protective metabolic syndrome biomarker. Its levels are inversely proportional to obesity, BMI, N-terminal prohormone of brain natriuretic peptide (NT-proBNP), triglyceridemia and insulin resistance. It increases with weight loss and with use of insulin-sensitizing drugs [9]. At the site of inflammation, adiponectin seems to have anti-inflammatory action. It inhibits production of pro-inflammatory cytokines such as TNF- $\alpha, I L-6, I L-10$, and IFN- $\gamma$; however, its secretion is down-regulated in response to pro-inflammatory cytokines and oxidative stress [8, 9, 25-30].

Taken together, we hypothesized that leptin and adiponectin through their wide range of molecular and immune properties may play a significant role in pathogenesis of systemic sclerosis. Although numerous studies have focused on circulating adiponectin and leptin levels in SSc patients, they brought inconclusive or contradictory results [2, 26, 31-42].

\section{Aim}

The aim of this study was to determine serum leptin and adiponectin levels in SSc patients according to healthy controls and to define possible correlations with clinical and laboratory profiles of SSc patients.

\section{Material and methods}

\section{Patients}

We recruited to the study 48 Caucasian female patients with SSc (aged 34-84 years, mean \pm SD: $62 \pm 10.6$ years), fulfilling the American College of Rheumatology (ACR) and/or EULAR classification criteria $[18,19]$. The control group consisted of 38 healthy subjects, matched with patients for sex, age, BMI and race (aged 36-88, $56.3 \pm 9.7$ years).

Patients and healthy controls were voluntarily recruited and informed consent was obtained from all participants. The study was approved by the Bioethics Committee. Patients with overlap syndromes, kidney disease, diabetes mellitus, thrombosis, metabolic syndrome/hyperlipidemia, pregnancy, neoplastic diseases and those with habitual cigarette smoking and alcohol drinking were excluded from the study.

\section{Laboratory assessment}

The material for the study was fasting peripheral blood drawn in the morning. The samples were allowed to clot for $30 \mathrm{~min}$ and centrifuged for $15 \mathrm{~min}$ at $1000 \times \mathrm{g}$. Obtained sera were stored at $-70^{\circ} \mathrm{C}$ immediately after collection till further analysis. Serum concentrations of leptin were measured using commercially available ELISA Kits (Quantikine ELISA Kit R\&D Systems, Minneapolis, MN, USA) according to the manufacturer's instructions. The concentration level of cytokine was calculated using 
an appropriate standard curve generated by the reader ELISA ELX 800 Bio-tek Instruments, USA.

\section{Clinical evaluations}

Skin thickness was evaluated using a modified Rodnan skin score (mRSS) [43]. According to the criteria proposed by LeRoy et al., patients were classified as having either limited cutaneous SSc (ICSSC) or diffuse cutaneous SSc (dcSSc) [44]. The disease duration was measured from the onset of the first symptom, other than Raynaud phenomenon (RP), consistent with SSc. The disease activity was assessed according to the European Scleroderma Study Group (ESCSG) disease activity score for SSC (Valentini disease activity index) as active or inactive [45]. Microvascular abnormalities such as digital ulcers and osteolysis of the distal phalanges of the fingers (acroosteolysis) were also evaluated. Nailfold capillaroscopy was performed to estimate SSc microangiopathy and patients were classified into three groups presenting as an early, active or late pattern, according to the criteria proposed by Cutolo et al. [46]. Patients were evaluated with respiratory function tests (forced vital capacity - FVC, total lung capacity - TLC and diffusing capacity of the lungs for carbon monoxide - DLCO) and high-resolution computed tomography (HRCT). Patients with ground glass opacification, centrilobular nodules or a honeycomb picture were considered to have lung involvement. Pulmonary artery pressure and valvular insufficiency were assessed by color Doppler echocardiography (ECO). Pulmonary arterial hypertension (PAH) was defined as systolic pulmonary arterial pressure (sPAP) $\geq 35 \mathrm{~mm} \mathrm{Hg}$ in Doppler echocardiography and was determined only at rest [47]. The presence of ANAs and their characteristics including anticentromere antibodies (ACAs), anti-topoisomerase 1 (anti-topo 1, Scl-70) antibodies, anti-RNA polymerase I or III antibodies, anti-U3- and U1-RNP, PM-SCl and anti-Ku antibodies was determined by means of indirect immunofluorescence on HEp-2 cells and/or immunoblot analysis.

\section{Statistical analysis}

The results were analyzed statistically using Statistica 10.0 PL software. Because the measurements were characterized by high skewness, the results were presented as the median, a measure of central tendency. Equality of distribution for each variable within normal distribution groups was tested using the Lilliefors version of the Kolmogorov-Smirnov test as well as the Shapiro-Wilk test. Because the test variables did not have a normal distribution, non-parametric tests were used for further analysis. These tests are resistant to deviations from the assumptions of normality of distribution and heterogeneity of variance in the groups compared. Pairs of independent groups were compared using the Mann-Whitney $U$ test. The relationship between leptin and patient characteristics were correlated by Spearman's rank correlation test, where $p<0.05$ was considered as statistically significant.

\section{Results}

Out of 48 patients, 42 had IcSSc and 6 had dcSSc. Mean disease duration was $12.85 \pm 7.63$ years. Limited

Table 1. Demographic and laboratory characteristics of patients with systemic sclerosis (SSc) and healthy control group

\begin{tabular}{|c|c|c|}
\hline Parameter & $\begin{array}{l}\text { Patient group } \\
\quad(n=48)\end{array}$ & $\begin{array}{l}\text { Control group } \\
(n=38)\end{array}$ \\
\hline \multirow[t]{2}{*}{ Age, mean $\pm S D$; range [years] } & $62.68 \pm 10.59 ; 34-84$ & $56.35 \pm 9.87 ; 36-88$ \\
\hline & \multicolumn{2}{|c|}{$Z=1.535, p=0.11$} \\
\hline Sex - female : male & $48: 0$ & $38: 0$ \\
\hline Disease duration, mean \pm SD [years] & $12.85 \pm 7.6$ & \\
\hline Duration of RP, mean \pm SD [years] & $16.91 \pm 9.3$ & \\
\hline \multirow[t]{2}{*}{$\mathrm{BMI}$, mean $\pm \mathrm{SD}\left[\mathrm{kg} / \mathrm{m}^{2}\right]$} & $26.57 \pm 4.42$ & $25.51 \pm 3.38$ \\
\hline & \multicolumn{2}{|c|}{$Z=1.347, p=0.177$} \\
\hline \multirow[t]{2}{*}{ Total cholesterol, mean \pm SD [mg/dl] } & $197.62 \pm 34.32$ & $202.06 \pm 30.27$ \\
\hline & \multicolumn{2}{|c|}{$Z=-0.634, p=0.52$} \\
\hline \multirow[t]{2}{*}{$\mathrm{LDL}$, mean $\pm \mathrm{SD}[\mathrm{mg} / \mathrm{dl}]$} & $115.12 \pm 28.07$ & $116.34 \pm 27.14$ \\
\hline & \multicolumn{2}{|c|}{$Z=-0.09, p=0.927$} \\
\hline \multirow[t]{2}{*}{$\mathrm{HDL}$, mean $\pm \mathrm{SD}[\mathrm{mg} / \mathrm{dl}]$} & $57.39 \pm 19.69$ & $62.79 \pm 12.80$ \\
\hline & \multicolumn{2}{|c|}{$Z=-1.895, p=0.058$} \\
\hline \multirow[t]{2}{*}{$\mathrm{TG}$, mean $\pm \mathrm{SD}[\mathrm{mg} / \mathrm{dl}]$} & $127 \pm 44.23$ & $104.36 \pm 35.40$ \\
\hline & \multicolumn{2}{|c|}{$Z=2.630, p=0.008$} \\
\hline
\end{tabular}

$R P$ - Raynaud phenomenon, BMI - body mass index, $L D L$ - low-density lipoprotein, $H D L$ - high-density lipoprotein, TG - triglycerides, SD - standard deviation. 
cutaneous SSc was diagnosed in 42 patients, whereas the diffuse disease subtype was diagnosed among 6 SSc patients. All of the patients manifested RP, whereas $13(27 \%)$ of them had active digital ulcers. When evaluated relative to the Valentini activity index, 9 (18.7\%) patients had active disease and 39 (81.25\%) had inactive disease. Furthermore, scleroderma-related interstitial lung disease (ILD), defined based on HRCT findings, was present in 43 (89.5\%) patients and 11 (22.9\%) SSc patients had elevated pulmonary artery pressure (SPAP $\geq 35 \mathrm{~mm} \mathrm{Hg}$ ) on ECO. Concerning cardiac involvement, 6 (12.5\%) SSc patients had ECG changes and 31 (64.5\%) had heart valves abnormalities. Considering the laboratory characteristics of SSc patients, the mean erythrocyte sedimentation rate (ESR) value in SSc patients was $26.065 \pm 15.6 \mathrm{~mm} / \mathrm{h}$ (range: $7-80 / \mathrm{h}$ ), and the mean C-reactive protein (CRP) concentration was $7.94 \pm 17.2 \mathrm{mg} / \mathrm{l}$ (range: 0.5-88.6). There was no significant difference in age, lipid profile values or BMI of SSc patients and those of the control group, except for higher TG values among the SSc group. Detailed demographic and laboratory characteristics of patients with SSc and the healthy control group are summarized in Table 1.

Considering the demographic characteristics of patients and the control group, there was no statistically significant difference in age $(p=0.11)$ and no statistically significant difference was found according to BMI ( $p=$ 0.177), total cholesterol, low-density lipoproteins (LDL) or high-density lipoprotein (HDL) (Table 1).

Leptin levels had a strong negative correlation with age in the patient group $(r=-0.507, p=0.000276)$ in contrast to healthy controls ( $p=0.258$ ), while there was a significant positive correlation between adiponectin values and age in SSc patients ( $r=0.478, p=0.000586)$ in contrast to controls ( $p=0.491)$. Consequently, leptin/ adiponectin ratio showed a strong inverse correlation in
SSc patients $(r=-0.583 ; p=0.000017)$ in contrast to healthy individuals $(r=-0.274 ; p=0.100)$.

There was a significant positive correlation between leptin levels and BMI only among SSc patients ( $r=0.495$; $p=0.000398)$; in contrast, adiponectin was strongly inversely correlated with $\mathrm{BMI}$ in the patient group $(r=-0.306 ; p=0.0342)$ in contrast to healthy controls $(p=0.256)$. Leptin/adiponectin ratio positively correlated with BMI in both groups; however, only in SSc patients a strong negative correlation was observed between leptin and adiponectin serum levels $(r=-0.314 ; p=$ 0.0312) (Table 2).

There was no significant change in either leptin or adiponectin levels in SSc patients as compared with healthy controls. Similarly, we found no significant differences in leptin/adiponectin, leptin/BMI and adiponectin/BMI ratios between the patient group and controls (Table 3).

\section{Association between leptin and adiponectin levels as well as their ratios and clinical characteristics of patients with SSC}

Diffuse form of the disease (dcSSc) was associated with significantly lower serum adiponectin levels $(8638.62 \pm 10382.62)$ and a close to significantly lower adiponectin/BMI ratio $(897.87 \pm 796.88)$ compared to patients with ICSSC (12425.92 $\pm 8615.65 ; p=0.042$ for adiponectin and 496.29 \pm 401.86 ; $p=0.059$ for adiponectin/BMI ratio) (Table 4). The values of adiponectin and adiponectin/BMI ratio were also significantly lower in patients with dcSSc compared with controls ( $p=0.048$ and $p=0.048$, respectively). No significant differences in analyzed parameters were found for patients with IcSSc.

Although lower serum leptin concentrations and lower leptin/BMI ratio were associated with anti-topol antibodies (20497.58 \pm 17745.13 and $786.42 \pm 664.61$, respectively) compared to ACA positive sera (42417.62

Table 2. Correlation of leptin and adiponectin levels as well as their ratio with age and BMI among patients and healthy controls (Spearman test)

\begin{tabular}{lcccc}
\hline Correlation & \multicolumn{2}{c}{ Patient group } & \multicolumn{2}{c}{ Control group } \\
\cline { 2 - 5 } & $R$ Spearman & $P$-value & $R$ Spearman & $P$-value \\
\hline Age: & & & & 0.258 \\
\hline Leptin & -0.507 & 0.000276 & -0.190 & 0.491 \\
\hline Adiponectin & 0.478 & 0.000586 & 0.116 & 0.100 \\
\hline Leptin/adiponectin ratio & -0.583 & 0.000017 & -0.274 & 0.070 \\
\hline BMI: & & & 0.296 & 0.256 \\
\hline Leptin & 0.495 & 0.000398 & 0.041 \\
\hline Adiponectin & -0.306 & 0.0342 & -0.188 & 0.332 \\
\hline Leptin/adiponectin ratio & 0.488 & 0.000496 & & 0.612 \\
\hline Leptin: & & & -0.084 & \\
\hline Adiponectin & -0.314 & 0.0312 & & \\
\hline
\end{tabular}

$B M I-$ body mass index 
Table 3. Leptin and adiponectin serum levels as well as leptin/adiponectin, leptin/BMI and adiponectin/BMI ratios in patients with SSc and control group (Mann-Whitney U-test)

\begin{tabular}{|c|c|c|}
\hline Parameter & $\begin{array}{l}\text { Patient group } \\
\quad(n=48)\end{array}$ & $\begin{array}{l}\text { Control group } \\
\quad(n=38)\end{array}$ \\
\hline \multirow[t]{2}{*}{ Leptin [pg/ml] } & $35025.02 \pm 37395.9$ & $36939.5 \pm 30594.97$ \\
\hline & \multicolumn{2}{|c|}{$Z=-0.852, p=0.393$} \\
\hline \multirow[t]{2}{*}{ Adiponectin [ng/ml] } & $11873.61 \pm 8874.70$ & $10803.4 \pm 5633.36$ \\
\hline & \multicolumn{2}{|c|}{$Z=-0.269, p=0.787$} \\
\hline \multirow[t]{2}{*}{ Leptin/adiponectin ratio } & $5.56 \pm 7.85$ & $4.63 \pm 5.53$ \\
\hline & \multicolumn{2}{|c|}{$Z=-0.163, p=0.870$} \\
\hline \multirow[t]{2}{*}{ Leptin/BMI } & $1242.22 \pm 1212.22$ & $1444.23 \pm 1222.64$ \\
\hline & \multicolumn{2}{|c|}{$Z=-0.989, p=0.322$} \\
\hline \multirow[t]{2}{*}{ Adiponectin/BMI } & $475.64 \pm 408.93$ & $435.27 \pm 241.58$ \\
\hline & \multicolumn{2}{|c|}{$Z=-0.547, p=0.583$} \\
\hline
\end{tabular}

$B M I-$ body mass index. Results are given as mean $\pm S D$.

Table 4. Comparison of serum adipocytokine levels and ratios in SSc patients according to disease subtype and profile of antinuclear antibodies (Mann-Whitney U-test)

\begin{tabular}{|c|c|c|}
\hline \multirow[t]{2}{*}{ Parameter } & \multicolumn{2}{|c|}{ SSc patient group } \\
\hline & $\operatorname{IcSSc}(n=42)$ & $\operatorname{dcSSc}(n=6)$ \\
\hline \multirow[t]{2}{*}{ Leptin, mean \pm SD $[\mathrm{pg} / \mathrm{ml}]$} & $36744.45 \pm 39159.91$ & $23275.56 \pm 20196.15$ \\
\hline & \multicolumn{2}{|c|}{$Z=0.733, p=0.463$} \\
\hline \multirow[t]{2}{*}{ Adiponectin, mean $\pm \mathrm{SD}[\mathrm{ng} / \mathrm{ml}]$} & $12425.92 \pm 8615.65$ & $8638.62 \pm 10382.62$ \\
\hline & \multicolumn{2}{|c|}{$Z=2.030, p=0.042$} \\
\hline \multirow[t]{2}{*}{ Leptin/adiponectin ratio } & $5.14 \pm 7.35$ & $8.45 \pm 11.08$ \\
\hline & \multicolumn{2}{|c|}{$Z=-0.223, p=0.823$} \\
\hline \multirow[t]{2}{*}{ Leptin/BMI } & $1292.61 \pm 1261.00$ & $897.87 \pm 796.88$ \\
\hline & \multicolumn{2}{|c|}{$Z=0.765, p=0.444$} \\
\hline \multirow[t]{3}{*}{ Adiponectin/BMI } & $496.29 \pm 401.86$ & $354.71 \pm 461.88$ \\
\hline & \multicolumn{2}{|c|}{$Z=1.884, p=0.059$} \\
\hline & ACA positive $(n=26)$ & Topol positive $(n=17)$ \\
\hline \multirow[t]{2}{*}{ Leptin, mean \pm SD $[\mathrm{pg} / \mathrm{ml}]$} & $42417.62 \pm 44716.42$ & $20497.58 \pm 17745.13$ \\
\hline & \multicolumn{2}{|c|}{$Z=1.838, p=0.066$} \\
\hline \multirow[t]{2}{*}{ Adiponectin, mean $\pm \mathrm{SD}[\mathrm{ng} / \mathrm{ml}]$} & $12984.13 \pm 8763.01$ & $10190.23 \pm 8241.17$ \\
\hline & \multicolumn{2}{|c|}{$Z=1.408, p=0.159$} \\
\hline \multirow[t]{2}{*}{ Leptin/adiponectin } & $5.651 \pm 8.378$ & $4.567 \pm 7.158$ \\
\hline & \multicolumn{2}{|c|}{$Z=0.894, p=0.371$} \\
\hline \multirow[t]{2}{*}{ Leptin/BMI } & $1473.83 \pm 1418.99$ & $786.42 \pm 664.61$ \\
\hline & \multicolumn{2}{|c|}{$Z=1.863, p=0.062$} \\
\hline \multirow[t]{2}{*}{ Adiponectin/BMI } & $521.26 \pm 427.88$ & $418.74 \pm 375.03$ \\
\hline & \multicolumn{2}{|c|}{$Z=1.002, p=0.316$} \\
\hline
\end{tabular}

SD - standard deviation, ICSSC - limited cutaneous systemic sclerosis, dcSSC - diffuse cutaneous systemic sclerosis, BMI - body mass index, ACA - anticentromere antibodies, Topol - anti-topo I (Scl-70) antibodies. Results are given as mean \pm SD.

\pm 44716.42 and $1473.83 \pm 1418.99$ ), the difference did not reach statistical significance $(p=0.066$ and $p=0.062$ respectively) (Table 4). However, compared to healthy controls, patients with anti-topol antibodies showed a significant decrease in serum leptin and leptin/BMI ratio ( $p=0.031$ and $p=0.026$ respectively).

Levels of serum leptin, adiponectin, leptin/adiponectin, leptin/BMI and adiponectin/BMI ratios were not as- 
Table 5. Correlation between leptin, adiponectin and leptin/adiponectin, leptin/BMI and adiponectin/BMI ratios with selected clinical and laboratory findings among SSc patients (Spearman's rank correlation test)

\begin{tabular}{lccccc}
\hline Parameter & Leptin & Adiponectin & $\begin{array}{c}\text { Leptin/ } \\
\text { Adiponectin }\end{array}$ & Leptin/BMI & Adiponectin/BMI \\
\hline ESR & $r=0.106$ & $r=0.156$ & $r=-0.139$ & $r=-0.119$ & $r=0.150$ \\
& $p=0.478$ & $p=0.286$ & $p=0.350$ & $p=0.424$ & $p=0.307$ \\
\hline CRP & $r=0.069$ & $r=-0.139$ & $r=0.129$ & $r=0.028$ & $r=-0.183$ \\
& $p=0.640$ & $p=0.345$ & $p=0.384$ & $p=0.851$ & $p=0.212$ \\
\hline mRSS & $r=-0.141$ & $r=-0.093$ & $r=-0.106$ & $r=-0.168$ & $r=-0.075$ \\
& $p=0.344$ & $p=0.525$ & $p=0.478$ & $p=0.258$ & $p=0.608$ \\
\hline Activity index & $r=-0.342$ & $r=-0.047$ & $r=-0.303$ & $r=-0.370$ & $r=-0.024$ \\
& $p=0.0185$ & $p=0.748$ & $p=0.037$ & $p=0.010$ & $p=0.868$ \\
\hline C3 & $r=0.537$ & $r=-0.049$ & $r=0.429$ & $r=0.512$ & $r=-0.108$ \\
& $p=0.00014$ & $p=0.745$ & $p=0.0032$ & $p=0.000318$ & $p=0.472$ \\
\hline C4 & $r=0.340$ & $r=0.046$ & $r=0.201$ & $r=0.302$ & $r=-0.035$ \\
& $p=0.022$ & $p=0.757$ & $p=0.183$ & $p=0.043$ & $p=0.817$ \\
\hline Disease duration [years] & $r=-0.225$ & $r=0.280$ & $r=-0.253$ & $r=-0.216$ & $r=0.295$ \\
& $p=0.128$ & $p=0.053$ & $p=0.085$ & $p=0.143$ & $p=0.041$ \\
\hline DLCO & $r=-0.278$ & $r=0.320$ & $r=-0.324$ & $r=-0.272$ & $r=0.345$ \\
& $p=0.177$ & $p=0.109$ & $p=0.113$ & $p=0.186$ & $p=0.083$ \\
\hline TLC & $r=0.163$ & $r=0.155$ & $r=0.076$ & $r=0.164$ & $r=0.137$ \\
& $p=0.334$ & $p=0.352$ & $p=0.652$ & $p=0.329$ & $p=0.408$ \\
\hline
\end{tabular}

ESR - erythrocyte sedimentation rate, CRP - C-reactive protein, mRSS - modified Rodnan skin score, BMI - body mass index, C3 - C3 complement concentration, C4 - C4 complement concentration, DLCO - diffusing capacity of the lungs for carbon monoxide, TLC - total lung capacity.

sociated with disease duration, inflammatory markers, ESR and CRP as well as total skin thickness score (mRSS); however, we found a close to significant positive correlation of disease duration with serum adiponectin levels as well as a weak significant correlation with adiponectin/ BMI ratio among studied patients with SSc $(r=0.280$; $p=0.053)$ (Table 5).

What is more, patients with a low C3 complement concentration had lower leptin levels (12026.28 $\pm 12734.57)$ than those with normal C3 values (40394.01 $\pm 39711.47 ; p=0.004)$ and leptin levels positively correlated with C3 concentrations $(R=0.537, p=0.00014)$. Leptin/BMI ratio and leptin/adiponectin ratio were both also significantly lower in SSc patients with lower C3 complement (488.84 \pm 567.18 and $2.00 \pm 2.71)$ compared to those with normal C3 values (1408.71 $\pm 1261.01 ; p=$ 0.005 and $6.56 \pm 8.63 ; p=0.034$, respectively) and C3 values positively correlated with leptin/adiponectin ratio $(r=0.429 ; p=0.0032)$. There were no significant differences when analyzed according to patients with normal or decreased C4 complement level; however, C4 concentrations showed a significant positive correlation with leptin levels ( $r=0.340 ; p=0.022$ ) (Table 6).

When considering organ involvement, adiponectin serum levels as well as values of adiponectin/BMI ratio were significantly lower in patients with decreased FVC values $(8598.92 \pm 11931.20$ and $318.33 \pm 443.35$, respectively) then in patients with normal FVC $(12592.19 \pm 7981.97$; $p=0.046$ and $493.06 \pm 349.35 ; p=0.038$, respectively).
Similarly, SSc patients with decreased TLC values had significantly lower adiponectin levels $(6815.72 \pm 4990.39)$ compared to SSc individuals with normal TLC (14102.66 $\pm 9494.23 ; p=0.018)$; although there was no significant correlation between serum adiponectin and TLC values $(r=0.155 ; p=0.352)$. We also did not observe any statistically significant associations between leptin and adiponectin levels or leptin/adiponectin, leptin/BMI and adiponectin/BMI ratios and the presence of ILD or PAH or decreased DLCO (Table 6).

Regarding clinical complication of SSc microangiopathy, we did not find any association between analyzed serum adipocytokine levels and the presence of active digital ulcers or nailfold videocapillaroscopy characteristics (data not shown).

A comparison made among patients with SSC stratified according to the activity of the disease showed leptin levels to be significantly lower in active patients than in those with inactive disease $(Z=-2.604$; $p=0.009$ ). Using Spearman's rank correlation test, leptin serum levels showed a strong negative correlation with score of disease activity index $(r=-0.342 ; p=0.018)$. Importantly, leptin/BMI ratio was also significantly lower in active patients $(420.18 \pm 502.73)$ then in those with inactive disease $(1410.84 \pm 1249.33$; $p=0.0032)$ and inversely correlated with the value of the activity index $(r=-0.370 ; p=0.010)$. Activity index score also negatively correlated with leptin/adiponectin ratio $(r=-0.303$; $p=0.037$ ) (Tables 5 and 6). 
Table 6. Comparison of serum adipocytokine levels and ratios among SSc patients according to pulmonary involvement, disease activity and C3 or C4 complement concentration (Mann-Whitney U-test)

\begin{tabular}{|c|c|c|c|c|}
\hline \multirow[t]{2}{*}{ Parameter } & \multicolumn{2}{|c|}{ Clinical parameter } & \multirow[t]{2}{*}{$z$} & \multirow[t]{2}{*}{$P$-value } \\
\hline & Active disease $(n=9)$ & Inactive disease $(n=39)$ & & \\
\hline Leptin [pg/ml] & $13700.49 \pm 18293.32$ & $39399.28 \pm 38933.9$ & -2.604 & 0.009 \\
\hline Adiponectin [ng/ml] & $8898.05 \pm 7925.16$ & $12560.27 \pm 9033.85$ & 1.571 & 0.116 \\
\hline Leptin/adiponectin & $2.80 \pm 3.31$ & $6.13 \pm 8.41$ & 1.556 & 0.119 \\
\hline Leptin/BMI & $420.18 \pm 502.73$ & $1410.84 \pm 1249.33$ & -2.943 & 0.0032 \\
\hline \multirow[t]{2}{*}{ Adiponectin/BMI } & $335.49 \pm 337.27$ & $507.98 \pm 420.90$ & 1.545 & 0.122 \\
\hline & ILD positive $(n=43)$ & ILD negative $(n=5)$ & & \\
\hline Leptin [pg/ml] & $36171.35 \pm 39340.75$ & $24544.53 \pm 10647.40$ & -0.155 & 0.876 \\
\hline Adiponectin [ng/ml] & $12264.97 \pm 9136.56$ & $9240.90 \pm 6370.34$ & -0.343 & 0.731 \\
\hline Leptin/adiponectin & $5.69 \pm 8.25$ & $4.31 \pm 3.61$ & 0.136 & 0.891 \\
\hline Leptin/BMI & $1270.60 \pm 1277.50$ & $941.7784 \pm 310.22$ & 0.077 & 0.937 \\
\hline \multirow[t]{2}{*}{ Adiponectin/BMI } & $489.45 \pm 422.92$ & $385.47 \pm 289.96$ & -0.381 & 0.703 \\
\hline & Decreased DLCO $(n=9)$ & DLCO in normal range $(n=17)$ & & \\
\hline Leptin $[\mathrm{pg} / \mathrm{ml}]$ & $41227.08 \pm 18774.04$ & $39879.45 \pm 47631.33$ & -1.103 & 0.269 \\
\hline Adiponectin [ng/ml] & $9726.34 \pm 5169.09$ & $14254.62 \pm 10440.89$ & 0.8354 & 0.403 \\
\hline Leptin/adiponectin & $7.34 \pm 8.63$ & $4.64 \pm 5.04$ & -1.132 & 0.257 \\
\hline Leptin/BMI & $1523.35 \pm 689.15$ & $1337.92 \pm 1279.72$ & -1.019 & 0.308 \\
\hline \multirow[t]{2}{*}{ Adiponectin/BMI } & $360.79 \pm 189.67$ & $603.41 \pm 522.28$ & 0.997 & 0.318 \\
\hline & Decreased FVC $(n=6)$ & FVC in normal range $(n=35)$ & & \\
\hline Leptin $[\mathrm{pg} / \mathrm{ml}]$ & $23815.10 \pm 18750.11$ & $37500.57 \pm 41336.66$ & 0.416 & 0.676 \\
\hline Adiponectin [ng/ml] & $8598.92 \pm 11931.20$ & $12592.19 \pm 7981.97$ & 1.991 & 0.046 \\
\hline Leptin/adiponectin & $9.21 \pm 10.57$ & $4.74 \pm 7.59$ & -1.022 & 0.306 \\
\hline Leptin/BMI & $890.79 \pm 754.87$ & $1311.29 \pm 1319.08$ & 0.568 & 0.569 \\
\hline \multirow[t]{2}{*}{ Adiponectin/BMI } & $318.33 \pm 443.35$ & $493.06 \pm 349.35$ & 2.065 & 0.038 \\
\hline & Decreased TLC $(n=12)$ & TLC in normal range $(n=26)$ & & \\
\hline Leptin $[\mathrm{pg} / \mathrm{ml}]$ & $21906.26 \pm 18329.76$ & $41698.69 \pm 44088.4$ & 1.115 & 0.264 \\
\hline Adiponectin [ng/ml] & $6815.72 \pm 4990.39$ & $14102.66 \pm 9494.23$ & 2.351 & 0.018 \\
\hline Leptin/adiponectin & $6.84 \pm 9.12$ & $5.39 \pm 8.22$ & -0.283 & 0.777 \\
\hline Leptin/BMI & $831.22 \pm 712.32$ & $1436.92 \pm 1404.22$ & 1.239 & 0.215 \\
\hline \multirow[t]{2}{*}{ Adiponectin/BMI } & $269.14 \pm 210.61$ & $563.21 \pm 450.10$ & 2.111 & 0.034 \\
\hline & PAH positive $(n=11)$ & PAH negative $(n=37)$ & & \\
\hline Leptin [pg/ml] & $26867.08 \pm 22572.98$ & $37361.81 \pm 41390.21$ & 0.437 & 0.661 \\
\hline Adiponectin [ng/ml] & $13066.65 \pm 10342.41$ & $11479.82 \pm 8635.04$ & -0.301 & 0.763 \\
\hline Leptin/adiponectin & $4.09 \pm 4.30$ & $6.09 \pm 8.76$ & 0.360 & 0.718 \\
\hline Leptin/BMI & $987.94 \pm 818.72$ & $1314.55 \pm 1327.62$ & 0.579 & 0.562 \\
\hline \multirow[t]{2}{*}{ Adiponectin/BMI } & $532.81 \pm 451.38$ & $458.79 \pm 406.22$ & -0.276 & 0.782 \\
\hline & C3 low level $(n=11)$ & C3 in normal range $(n=37)$ & & \\
\hline Leptin [pg/ml] & $12026.28 \pm 12734.57$ & $40394.01 \pm 39711.47$ & 2.880 & 0.004 \\
\hline Adiponectin [ng/ml] & $14512.23 \pm 12300.93$ & $11023.30 \pm 7715.43$ & -0.351 & 0.725 \\
\hline Leptin/adiponectin & $2.00 \pm 2.71$ & $6.56 \pm 8.63$ & 2.117 & 0.034 \\
\hline Leptin/BMI & $488.85 \pm 567.18$ & $1408.71 \pm 1261.01$ & 2.796 & 0.005 \\
\hline Adiponectin/BMI & $429.29 \pm 337.22$ & $623.12 \pm 596.72$ & -0.502 & 0.615 \\
\hline
\end{tabular}


Table 6. Cont. Comparison of serum adipocytokine levels and ratios among SSc patients according to pulmonary involvement, disease activity and C3 or C4 complement concentration (Mann-Whitney U-test)

\begin{tabular}{|c|c|c|c|c|}
\hline \multirow[t]{2}{*}{ Parameter } & \multicolumn{2}{|c|}{ Clinical parameter } & \multirow[t]{2}{*}{$z$} & \multirow[t]{2}{*}{$P$-value } \\
\hline & C4 low level $(n=4)$ & C4 in normal range $(n=44)$ & & \\
\hline Leptin [pg/ml] & $14910.16 \pm 19968.36$ & $36066.83 \pm 38293.32$ & 1.481 & 0.138 \\
\hline Adiponectin [ng/ml] & $19582.07 \pm 15559.86$ & $11119.65 \pm 8033.81$ & -1.029 & 0.303 \\
\hline Leptin/adiponectin & $2.93 \pm 5.12$ & $5.82 \pm 8.15$ & 1.305 & 0.191 \\
\hline Leptin/BMI & $581.44 \pm 805.26$ & $1268.48 \pm 1224.77$ & 1.481 & 0.138 \\
\hline Adiponectin/BMI & $884.72 \pm 829.69$ & $436.51 \pm 347.00$ & -0.991 & 0.321 \\
\hline
\end{tabular}

BMI - body mass index, C3 - C3 complement concentration, C4 - C4 complement concentration, DLCO - diffusing capacity of the lungs for carbon monoxide, $T L C$ - total lung capacity, ILD - interstitial lung disease, PAH - pulmonary arterial hypertension, FVC - forced vital capacity. Results are given as mean \pm SD.

Table 7. Comparison of important inflammatory and clinical markers between patients with active and inactive disease (Mann-Whitney $U$ test)

\begin{tabular}{lcccc}
\hline Parameter & Active disease & Inactive disease & $\boldsymbol{Z}$ & $P$-value \\
\hline ESR $[\mathrm{mm} / \mathrm{h}]$ & $28.22 \pm 13.91$ & $24.33 \pm 16.00$ & -1.109 & 0.267 \\
\hline CRP $[\mathrm{mg} / \mathrm{ml}]$ & $15.31 \pm 27.96$ & $6.24 \pm 13.61$ & -0.832 & 0.405 \\
\hline C3 & $0.99 \pm 0.24$ & $1.12 \pm 0.19$ & 1.675 & 0.09 \\
\hline C4 & $0.22 \pm 0.08$ & $0.24 \pm 0.08$ & 0.969 & 0.332 \\
\hline mRSS & $13.22 \pm 7.39$ & $9.25 \pm 5.77$ & -1.386 & 0.165 \\
\hline BMI & $28.59 \pm 4.24$ & $26.10 \pm 4.38$ & -1.518 & 0.128 \\
\hline
\end{tabular}

ESR - erythrocyte sedimentation rate, CRP - C-reactive protein, C3-C3 complement concentration, C4 - C4 complement concentration, mRSS - modified Rodnan skin score, $B M I-$ body mass index. Results are given as mean $\pm S D$.

Importantly, we found no significant difference between the active and inactive patients relative to mean BMI values $\left(28.59 \pm 4.24 \mathrm{~kg} / \mathrm{m}^{2}\right.$ vs. $26.10 \pm 4.38 \mathrm{~kg} / \mathrm{m}^{2} ; p=$ 0.128). Moreover, there was no statistically significant difference between the active and inactive patients in terms of serum inflammatory markers, ESR $(28.22 \pm 13.91 \mathrm{vs}$. $24.33 \pm 16.00 \mathrm{~mm} / \mathrm{h} ; p=0.267)$ or CRP $(15.31 \pm 27.96 \mathrm{vs}$. $6.24 \pm 13.61 \mathrm{~mm} / \mathrm{h} ; p=0.405)$. Similarly, the mRSS score did not differ in patients with active SSc (13.22 \pm 7.39$)$ compared to those with inactive disease $(9.25 \pm 5.77 ; p=$ 0.165). Although C3 levels were lower in active patients, the difference was not statistically significant $(p=0.09)$ (Table 7).

\section{Discussion}

In recent years, there are more and more data on the role of adipocytokines in autoimmune responses. Particularly, the role of leptin as a potent pro-inflammatory agent has been investigated in spontaneous models of autoimmunity and studies in humans have further delineated the role of leptin in the pathogenesis of autoimmune diseases such as diabetes, inflammatory bowel disease, Behçet's disease, systemic lupus erythematosus (SLE), and rheumatoid arthritis (RA) [3-5, 14, 18, 48-54]. Adiponectin, another active hormone released by adipocytes, seems to be an attractive opposite in the field of autoimmune diseases, since it is considered to act as an anti-inflammatory molecule, and recently some studies demonstrated that plasma levels of adiponectin in patients with autoimmune diseases might be related to inflammatory status in their pathogenesis [2, 6, 52, 55-57].

Although several studies have explored the role of circulating leptin and adiponectin levels in SSc patients, they gave inconclusive or contradictory results, and there are only rare reports that have analyzed both adipocytokines concomitantly.

Our study sought to compare serum leptin and adiponectin levels in SSc patients according to healthy controls and to determine whether leptin and adiponectin are associated with clinical profiles of the patients. Since leptin and adiponectin seem to have an opposing action in inflammatory and immune responses, we aimed to assess whether their abnormal ratio (leptin/adiponectin; L/A) rather than isolated values might be associated with disease manifestations. Moreover, since many factors, including gender, age and BMI, could influence adiponectin and leptin levels in serum, all patients and subjects from the control group were females adjusted for age, and did not differ significantly in BMI or lipid profile, except for higher triglycerides (TG) values among SSc patients. The analyzed laboratory data were adjusted to these demographic parameters to facilitate comparison of the evaluated groups (patients and controls). 
In our study, there was no significant difference of leptin and adiponectin levels in sera of SSc patients as compared to healthy controls or of leptin/adiponectin ratio between these groups; however, in SSc patients only a strong negative correlation was observed between leptin and adiponectin serum levels. This is in agreement with two recent meta-analyses - one of fourteen papers (six for serum leptin, six for serum adiponectin and two for both) performed by Zhao et al. [26] and the second of eleven studies (511 SSc patients and 341 controls) by Lee et al. [31] that showed no significant differences in serum leptin levels between SSc patients and healthy controls. However, in contrast to our observation, both meta-analyses revealed significantly lower serum adiponectin levels in SSc patients than in normal controls, but neither the leptin/adiponectin ratio nor a correlation between leptin and adiponectin levels was reported by those authors. When considering respective literature data concerning either serum leptin or adiponectin levels in SSc, they are inconsistent. Our results are in agreement with data from two previous studies by OlewiczGawlik et al. [32] and Budulgan et al. [33], who observed only slightly lower leptin levels in SSc patients than in healthy subjects, though not down to significant levels, and found no statistically significant differences in serum adiponectin. Similar to our report, serum adiponectin levels were also comparable between the SSc group and controls in the studies of other authors [34-36]. However, Kotulska et al. [37] as well as Winsz-Szczotka et al. [38, 39] reported a significantly lower serum leptin level in SSc patients, whereas the results of two recent studies detected significantly higher levels of circulating leptin in SSc subjects with respect to healthy controls [2, 40]. Although the present studies did not compare adiponectin and leptin levels in SSc with those in other autoimmune diseases, studies in RA and SLE have shown that these disorders are generally associated with elevated serum adiponectin or leptin [58-60]. Thus, our observations might indicate that, in contrast, SSc is not associated with abnormal levels of measured adipocytokines, despite the chronic inflammation that is thought to be a hallmark of this disease $[36,61]$.

These discrepancies between particular studies might result from the heterogeneity of SSc patients according to demographic parameters such as age, gender, BMI as well as the clinical profile of the disease or immunosuppressive treatment used.

Body mass index is one of the most potent factors influencing serum adipocytokine levels. In fact, in our study there was a strong positive correlation between either leptin levels or leptin/adiponectin ratio and BMI but only among SSc patients and, in contrast, adiponectin concentration was strongly inversely correlated with BMI in the patient group but not in healthy controls. Similarly, Kotulska et al. [37] reported a significant positive correlation of serum leptin with BMI only in SSC patients. This is in agreement with the notion that a relationship between BMI and leptin levels is not observed for normal individuals [62]. The authors indicated that a decreased serum leptin level and BMI may be a consequence of gastrointestinal tract involvement and low energy status with decreased fat stores and fasting in SSc patients since the lowest leptin levels were found in patients with more advanced disease [37]. In fact, in the study of Budulgan et al. [33], BMI values as well as leptin levels were significantly lower in the patient group than in controls and, similarly, Toussirot et al. [63] and Sari et al. [64] noted a possible relationship between reduced leptin levels and low BMI values. In patients with SSc, fat mass distribution may be different to that in controls. In particular, patients with SSc display lower subcutaneous adipose tissue accumulation than controls despite having similar BMI, as was also observed in RA patients [65]. This is attributed to skin fibrosis, and attrition of dermal white adipose tissue (dWAT) has been reported to correlate with reduced levels of circulating adiponectin in SSc patients [66]. This is in favor of our results; however, we did not evaluate fat stores or the loss of dWAT in our patients.

Moreover, disease duration has been reported to have an effect on serum adiponectin and leptin levels [67]. In fact, we observed a positive, close to significant correlation between serum adiponectin and the years from first non-Raynaud manifestation of the disease, and this correlation reached statistical significance when we analyzed adiponectin/BMI ratio. In favor of these results is the observation reported by Lakota et al. [36], who found low adiponectin levels in patients with disease in a relatively early stage (arbitrarily defined as fewer than 18 months from the first non-Raynaud manifestation) in comparison with patients with disease in the late stage (defined as more than 36 months). In contrast, the levels of adiponectin did not correlate with disease duration of investigated patients in the study by Olewicz-Gawlik et al. [32]. On the other hand, we did not find a significant correlation of leptin, L/A ratio and leptin/BMI according to disease duration, which is in agreement with data from previous studies [2, 37, 40]. In contrast, WinszSzczotka et al. as well as Olewicz-Gawlik et al. [32] reported a significant positive correlation between leptin and disease duration [38], but an inverse correlation of both leptin and adiponectin with the duration of the first non-RP symptom has also been reported [39]. Such conflicting results might be a consequence of different disease progression rate and severity in the studied group of SSc patients.

In fact, additionally to the role in inflammation, opposite action of leptin and adiponectin has been described in the field of connective tissue remodeling and fibrosis. Leptin seems to be a profibrogenic molecule, whereas adiponectin has been found to have antifibrogenic action [66, 68-72]. Profibrotic effects of leptin have been 
reported in bleomycin-induced lung fibrosis in mice by augmentation of transforming growth factor- $\beta$ (TGF- $\beta$ ) signaling, and in liver fibrosis, leptin acts as an activator of hepatic stellate cells (HSCs) and stimulates the transcriptional activation of collagen I and tissue inhibitor of metalloproteinases 1 (TIMP1) [71, 73, 74]. In contrast, adiponectin might inhibit the collagen gene expression and myofibroblast differentiation [70].

The most distinctive feature of tissue fibrosis in SSc is skin thickening and pulmonary interstitial fibrosis [61]. Significantly, recent studies demonstrated that adiponectin levels are reduced in patients with diffuse skin involvement and correlate with disease severity $[36,41]$. In general, our findings are in agreement with these suggestions. When considering disease subtype, diffuse form (dcSSc) was associated with significantly lower serum adiponectin levels and a close to significant decrease in adiponectin/BMI ratio compared to patients with limited subtype (ICSSC). Moreover, stratification by disease subtype revealed that both adiponectin and adiponectin/BMI ratio were significantly lower in the diffuse SSc group, but not the limited SSC group, than in controls. Although the results indicate a weak association, and should be treated with caution, this finding is in harmony with antifibrotic functions of this adipocytokine, and they are concordant with the data of recent studies. In particular, this is in agreement with results from the meta-analysis by Lee et al. [31] which revealed that the adiponectin level was significantly lower in the diffuse SSc group, but not the limited SSc group, than in the controls. This is also in line with the observation of Masiu et al. [34] and Lakota et al. [36] as well as Arakawa et al. [41]. In addition, in the study of Arakawa et al. [41], adiponectin mRNA levels in skin tissues from patients with dcSSc were also reduced. On the other hand, Olewicz-Gawlik et al. [32] did not observe statistically significant differences in adiponectin serum concentrations between dcSSc and lcSSc subgroups.

The difference in adiponectin levels between patients with IcSSc and those with dcSSc suggests that adiponectin might be associated with the severity of skin fibrosis. However, we did not find any correlation between either leptin or adiponectin and the value of total skin thickness score assessed by mRSS. Similarly, Pehlivan et al. and Budulgan et al. [2, 33] found no association between leptin levels and skin score in the SSc patients, but in the earlier studies, a negative correlation was observed, despite similar adiponectin serum levels between patients with SSc and healthy controls [35, 36, 41]. Moreover, Lakota et al. [36] reported a significant correlation between adiponectin mRNA expression in the skin and mRSS in the individual biopsies. This discrepancy might result from the different proportion of disease subtypes among SSc patients in particular reports, since in our study only 6 from 48 patients had dcSSc, while in those that reported a significant correlation, the ratio
dcSSc : IcSSc patients was higher: $13: 19$ according to Arakawa et al. [41] and 50 : 79 according to Lakota et al. [36]. In favor of this suggestion is that in the study of Masiu et al. [34] tissue levels of adiponectin were shown to inversely correlate with skin scores only in patients with diffuse SSC $(n=32)$ in contrast to those with limited subtype $(n=28)$.

There is convincing literature evidence of the role of low adiponectin in the pathogenesis of tissue fibrosis associated with SSc [36, 41, 66, 70]. In fibrosis of SSc, myofibroblast activation and collagen production are the most important events. Fang et al. [70] found that adiponectin could inhibit collagen gene expression and myofibroblast differentiation and demonstrated the anti-fibrotic effects of adiponectin in normal and scleroderma fibroblasts. They further demonstrated that the levels of adiponectin as well as its receptor are impaired in scleroderma patients. In addition, Lakota et al. [36] suggested that reduced adiponectin levels might reflect reduced peroxisome proliferatoractivated receptors- $\gamma$ (PPAR- $\gamma$ ) activity associated with active or progressive skin fibrosis. It has been reported that adiponectin production is tightly regulated by PPAR- $\gamma$ and, in turn, the anti-fibrotic effects of PPAR- $\gamma$ are partially mediated by adiponectin $[36,66]$. Moreover, in mice models lacking adiponectin an exaggerated dermal fibrotic response has been observed, while transgenic mice with constitutively elevated adiponectin showed protection from skin and peritoneal fibrosis. Importantly, adiponectin receptor agonists abrogated ex vivo fibrotic responses and prevented and reversed experimentally induced organ fibrosis in mice [66]. These results implicate aberrant adiponectin pathway activity in skin fibrosis, identifying a novel function for this pleiotropic adipokine in regulation of tissue remodeling. Thus, adiponectin may become a new focus for studies of the pathogenesis of SSc. More experiments are needed to confirm these results. Restoring adiponectin signaling in SSc patients therefore might represent an innovative pharmacological strategy for intractable organ fibrosis [66].

In contrast to adiponectin, we did not find a statistical association between leptin levels and disease subtype. Similar to our results, other authors did not find a significant relationship between mean leptin levels and disease subtype [2, 32, 40].

Leptin and adiponectin, and their respective receptors, are also expressed in the human lung [38, 39, 69]. However, in contrast to disease subtype, we did not find an association of serum leptin and adiponectin with radiological evidence of lung fibrosis (ILD) on HRCT scans, although, when lung function tests were assessed, significantly lower adiponectin levels as well as adiponectin/ $\mathrm{BMI}$ ratio characterized patients with restriction as assessed by decreased lung FVC and TLC values ( $<80 \%$ of normal value). Furthermore, no association was noted in our patients between serum adipocytokines and decreased DLCO. Our observations are in line with those 
of Olewicz-Gawlik et al. [32], who did not find a relationship between circulating adiponectin and radiological and functional parameters of lung involvement except VC. Also, in the study of Lakota et al. [36] neither FVC nor DLCO or radiologic evidence of pulmonary fibrosis could be demonstrated to have a significant correlation with serum adiponectin levels, although serum adiponectin correlated with the skin score. However, Masui [42] reported significantly decreased serum adiponectin levels in SSc patients with active ILD before cyclophosphamide pulses compared with healthy controls and a significant increase in adiponectin levels after completion of treatment, which correlated with the decrease in ILD scores. The reason for the lack of relationship between adiponectin and pulmonary parameters found in our study may be the fact that significant lung functional impairment and lung fibrosis on HRCT coexisted in a limited number of SSc patients (only 3 patients did not have ILD while decreased FVC and/or TLC were found in 7 and 13 patients, respectively). However, we cannot exclude the influence of immunosuppressive therapy or a bias caused by the limited total number of patients in our study.

The correlation of serum adiponectin levels with skin but not lung fibrosis points to potential differences in the pathomechanisms underlying fibrosis in these organs, as suggested by Lakota et al. [36]. Against this suggestion is the observation by Arakawa et al. [41] that SSc patients with decreased serum adiponectin levels had both a higher total skin thickness score and higher incidence of pulmonary fibrosis.

The second important finding of our study is a statistically significant negative correlation between serum leptin levels and the value of the disease activity index. Patients with active disease had significantly lower leptin concentrations than those with inactive SSc. Similar results were obtained by Budulgan et al. [33]. However, Pehlivan et al. [2] found that there was no association between leptin levels and disease activity. Importantly, in our study this negative correlation remained statistically significant when leptin to BMI ratio was analyzed, thus indicating that the association between leptin and disease activity might be independent of body mass index. Moreover, stratification by disease activity revealed that leptin levels as well as leptin/BMI were significantly lower in active, but not in inactive patients, than in controls, although these parameters where comparable between the total SSc group and healthy subjects. On the other hand, adiponectin levels as well as adiponectin/ $\mathrm{BMI}$ and leptin/adiponectin ratio were not found to have a significant correlation with disease activity, which is in contrast to the observation of Olewicz-Gawlik et al. [32], who reported significantly lower adiponectin concentrations in patients with active disease and a strong negative correlation between adiponectin level and the valentini activity index score.
The inverse correlation between leptin and disease activity as observed in our study might be somewhat intriguing since leptin is assumed to be a strong proinflammatory agent increasing the synthesis of pro-inflammatory cytokines such as IL- $6, \mathrm{IL}-12$ and TNF- $\alpha$ [ 6 , 8-11, 13]. In fact, in the literature there are some data on a strong positive correlation between leptin levels and disease activity in other rheumatic diseases, including SLE [4, 49-52, 58], RA [53, 75-77] and ankylosing spondylitis (AS) $[64,78]$, but to date the clinical significance of this elevation remains unknown. It is thought that serum leptin levels may be either a contributing factor or a marker of disease activity [14, 49, 54]. However, recent studies have suggested that leptin has a dual role in inflammation since it might express certain anti-inflammatory properties by releasing IL1 receptor antagonist (IL-1Ra) and increasing the production of anti-inflammatory IL-4 [13-15, 79, 80]. Furthermore, lower leptin levels in active patients might be explained by the mechanism that in the chronic inflammatory process, leptin is inhibited by long-acting pro-inflammatory cytokines in adipose tissue [17]. In fact, serum leptin level has been shown to have a negative correlation with inflammatory markers such as CRP and IL- 6 in patients with RA and prolonged in vitro stimulation of adipose tissue with TNF- $\alpha$ inhibits the production of leptin and leptin mRNA [81]. It may also be possible that leptin release is reduced during the active period and its anti-inflammatory influence is decreased.

For better evaluation of the association between leptin levels and disease activity, we further analyzed particular parameters, including systemic markers of inflammation, that can influence the activity index score. In this study, we found no correlation between either acute phase reactant CRP or ESR and leptin levels and, furthermore, there was no difference between the active and inactive patients with regard to CRP and ESR, which is in agreement with the previous observations of Budulgan et al. [33]. Moreover, lower leptin levels in active patients might be associated with lower BMI; however, in our study, we did not find a significant difference between active and inactive patients in terms of BMI. What is more, active and inactive patients did not differ according to skin involvement. However, we observed a significantly lower C3 complement level in patients with active disease as well as a strong, statistically significant positive correlation between serum leptin levels as well as leptin/BMI ratio and either C3 or C4 complement levels in SSc patients. Similar results concerning the association between leptin levels and C3 have been obtained by other authors [32, 33, 37]. Although adiponectin levels were not related to the concentrations of either $\mathrm{C} 3$ or C4 complement concentrations, leptin/adiponectin ratio was decreased in patients with lower C3 status and showed a strong positive correlation with C3. 
Since low C3 and C4 status may indicate endothelium activation or damage, our observations might suggest that lower leptin levels and leptin/adiponectin ratio in SSc patients with more active disease reflect vascular homeostasis rather than inflammation. In fact, leptin receptors are expressed on vascular endothelial cells and smooth muscle cells and leptin facilitate proliferation of human endothelial cells (ECS), supporting angiogenesis and neovascularization [82-84]. In contrast to leptin, adiponectin may play a role in the inflammatory vascular response by inhibiting the expression of adhesion molecules such as VCAM-1, ICAM-1 and E-selectins on endothelial cells, preventing activation and migration of immune cells [85]. Although there are no data concerning leptin or adiponectin levels in relation to aspects of microangiopathy in SSc patients, it is noteworthy that lower median plasma levels of adiponectin were found in subjects with diabetic foot as well as a significant negative correlation between adiponectin and diabetic retinopathy, which suggest a possible role of hypoadiponectinemia in microvascular involvement [86]. Nevertheless, we did not find a statistical association between leptin or adiponectin levels and microvascular complications in our SSc patients. Specifically, neither leptin nor adiponectin showed a significant difference between patients with digital ulcers and those without.

\section{Conclusions}

The results of our study indicate that, in contrast to other autoimmune diseases, SSc does not seem to be associated with abnormal levels of leptin or adiponectin, despite the chronic inflammation. However, according to our results, leptin and adiponectin might be differentially expressed in SSC sera and might be associated with different disease manifestations. In particular, we observed that lower adiponectin serum level as well as adiponectin/BMI ratio are associated with more severe skin involvement as well as with restriction on pulmonary function tests, and this finding is in harmony with its antifibrotic action, whereas leptin levels inversely correlated with disease activity index. Decreased serum leptin was associated with greater disease activity, which is somewhat intriguing in the face of its expected strong pro-inflammatory action. In this aspect, since we did not find a correlation between serum leptin and systemic markers of inflammation such as CRP and ESR which can influence the activity index score in SSc patients, it might be supposed that leptin's association with disease activity is independent of its contribution to inflammatory processes, which is in contrast to other rheumatic diseases. Instead, our study showed that lower serum leptin levels as well as decreased leptin/adiponectin ratio were related to a low status of complement component C3 or C4, thus suggesting that the association of leptin deficiency with higher disease activity reflects vascular status rather than systemic inflammation.

However, it has to be noted that our study has several limitations. The first of them is the small and homogeneous sample. Another potential drawback of the study may be a selection bias. Thus, further research enrolling larger groups of SSc patients as well as comparative studies with other rheumatic diseases such as SLE or RA seem to be justified.

Systemic sclerosis does not seem to be associated with abnormal levels of leptin or adiponectin, despite the chronic inflammation. Leptin and adiponectin are differentially expressed in SSc sera and might be associated with different disease manifestations. Low adiponectin status seems to be associated with intensity of cutaneous and pulmonary repercussions of tissue fibrosis in SSc. Decreased leptin levels might be related to more active disease, in particular reflecting vascular status in SSc patients.

\section{Conflict of interest}

The authors declare no conflict of interest.

\section{References}

1. Furue M, Mitoma C, Mitoma H, et al. Pathogenesis of systemic sclerosis-current concept and emerging treatments. Immunol Res 2017; 65: 790-7.

2. Pehlivan Y, Onat AM, Ceylan N, et al. Serum leptin, resistin and TNF-alpha levels in patients with systemic sclerosis: the role of adipokines in scleroderma. Int J Rheum Dis 2012; 15: 374-9.

3. Vadacca M, Margiotta DPE, Navarini L, Afeltra A. Leptin in immuno-rheumatological diseases. Cell Mol Immunol 2011; 8: 203-12.

4. Margiotta DPE, Vadacca M, Navarini L, et al. The complex role of leptin in SLE: is leptin a key link between metabolic syndrome, accelerated atherosclerosis and autoimmunity? Lupus Open Access 2016; 1: 107.

5. Cojocaru M, Cojocaru IM, Siloşi I, Rogoz S. Role of leptin in autoimmune diseases. Maedica (Buchar) 2013; 8: 68-74.

6. Stofkova A. Leptin and adiponectin: from energy and metabolic dysbalance to inflammation and autoimmunity. Endocr Regul 2009; 43: 157-68.

7. Tilg H, Moschen AR. Adipocytokines: mediators linking adipose tissue, inflammation and immunity. Nat Rev Immunol 2006; 6: 772-83.

8. Šenolt L. Adipokines: role in local and systemic inflammation of rheumatic diseases. Expert Rev Clin Immunol 2017; 13: 1-3.

9. Carbone F, La Rocca C, Matarese G. Immunological functions of leptin and adiponectin. Biochimie 2012; 94: 2082-8.

10. Procaccini C, Jirillo E, Matarese G. Leptin as an immunomodulator. Mol Aspects Med 2012; 33: 35-45.

11. Procaccini C, Pucino V, Mantzoros CS, Matarese G. Leptin in autoimmune diseases. Metabolism 2015; 64: 92-104.

12. Antuna-Puente B, Feve B, Fellahi S, Bastard JP. Adipokines: the missing link between insulin resistance and obesity. Diabetes Metab 2008; 34: 2-11. 
13. Paz-Filho G, Mastonardi C, Franco CB, et al. Leptin: molecular mechanisms, systemic pro-inflammatory effects, and clinical implications. Arq Bras Endocrinol Metabol 2012; 56: 597-607.

14. Matarese G, Moschos S, Mantzoros CS. Leptin in immunology. J Immunol 2005; 174: 3137-42.

15. Tian G, Liang JN, Wang ZY, Zhou D. Emerging role of leptin in rheumatoid arthritis. Clin Exp Immunol 2014; 177: 557-70.

16. Otero M, Lago R, Lago F, et al. Leptin, from fat to inflammation: old questions and new insights. FEBS Lett 2005; 579: 295-301.

17. Bruun JM, Pedersen SB, Kristensen K, Richelsen B. Effects of pro-inflammatory cytokines and chemokines on leptin production in human adipose tissue in vitro. Mol Cell Endocrinol 2002; 190: 91-9.

18. Brzeska A, Świdrowska J, Smolewska E. Leptin as an important hormone and immunomodulator in adult and childhood rheumatoid arthritis. Global J Immunol Allergic Dis 2013; 1: 16-24.

19. Batra A, Okur B, Glauben R, et al. Leptin: a critical regulator of CD4+ T-cell polarization in vitro and in vivo. Endocrinology 2010; 151: 56-62.

20.Fernández-Riejos P, Najib S, Santos-Alvarez J, et al. Role of leptin in the activation of immune cells. Mediators Inflamm 2010; 2010: 568343.

21. Agrawal S, Gollapudi S, Su H, Gupta S. Leptin activates human B cells to secrete TNF-alpha, IL-6, and IL-10 via JAK2/ STAT3 and p38MAPK/ERK1/2 signaling pathway. J Clin Immunol 2011; 31: 472-8.

22. Lord GM, Matarese G, Howard JK, et al. Leptin modulates the T-cell immune response and reverses starvation-induced immunosuppression. Nature 1998; 394: 897-901.

23. Busso N, So A, Chobaz-Peclat V, et al. Leptin signaling deficiency impairs humoral and cellular immune responses and attenuates experimental arthritis. J Immunol 2002; 168: 875-82.

24. Ehling A, Schäffler A, Herfarth $\mathrm{H}$, et al. The potential of adiponectin in driving arthritis. J Immunol 2006; 176: 4468-78.

25. Kadowaki T, Yamauchi T. Adiponectin and adiponectin receptors. Endocr Rev 2005; 26: 439-51.

26.Zhao JH, Huang XL, Duan Y, et al. Serum adipokines levels in patients with systemic sclerosis: a meta-analysis. Modern Rheumatol 2017; 27: 298-305.

27. Ouchi N, Walsh K. Adiponectin as an anti-inflammatory factor. Clin Chim Acta 2007; 380: 24-30.

28. Wilk S, Scheibenbogen C, Bauer S, et al. Adiponectin is a negative regulator of antigen-activated T cells. Eur J Immunol 2011; 41: 2323-32.

29. Fantuzzi G. Adiponectin in inflammatory and immune-mediated diseases. Cytokine 2013; 64: 1-10.

30. Ebrahimi-Mamaeghani M, Mohammadi S, Arefhosseini SR, et al. Adiponectin as a potential biomarker of vascular disease. Vasc Health Risk Manag 2015; 11: 55-70.

31. Lee YH, Song GG. Meta-analysis of circulating adiponectin, leptin, and resistin levels in systemic sclerosis. Z Rheumatol 2017; 76: 789-97.

32. Olewicz-Gawlik A, Dańczak-Pazdrowska A, Kuźnar-Kamińska B, et al. Circulating adipokines and organ involvement in patients with systemic sclerosis. Acta Reumatol Port 2015; 40: 156-62.

33. Budulgan M, Dilek B, Dağ ŞB, et al. Relationship between serum leptin level and disease activity in patients with systemic sclerosis. Clin Rheumatol 2014; 33: 335-9.

34. Masui Y, Asano Y, Shibata S, et al. Serum adiponectin levels inversely correlate with the activity of progressive skin scle- rosis in patients with diffuse cutaneous systemic sclerosis. J Eur Acad Dermatol Venereol 2012; 26: 354-60.

35. Tomčik M, Arima K, Hulejová H, et al. Adiponectin relation to skin changes and dyslipidemia in systemic sclerosis. Cytokine 2012; 58: 165-8.

36. Lakota K, Wei J, Carns M, et al. Levels of adiponectin, a marker for PPAR-gamma activity, correlate with skin fibrosis in systemic sclerosis: potential utility as biomarker? Arthritis Res Ther 2012; 14: R102.

37. Kotulska A, Kucharz EJ, Brzezińska-Wcisło L, Wadas U. A decreased serum leptin level in patients with systemic sclerosis. Clin Rheumatol 2001; 20: 300-2.

38. Winsz-Szczotka K, Kuźnik-Trocha K, Komosińska-Vassev K, Olczyk K. The serum level of leptin in systemic sclerosis patients. Ann Acad Med Siles 2011; 65: 42-8.

39. Winsz-Szczotka K, Kuźnik-Trocha K, Komosińska-Vassev K, et al. Relationship between adiponectin, leptin, IGF-1 and total lipid peroxides plasma concentrations in patients with systemic sclerosis: a possible role in disease development. Int J Rheum Dis 2016; 19: 706-14.

40. Riccieri V, Stefanantoni K, Vasile M, et al. Abnormal plasma levels of different angiogenic molecules are associated with different clinical manifestations in patients with systemic sclerosis. Clin Exp Rheumatol 2011; 29 (2 Suppl 65): S46-52.

41. Arakawa H, Jinnin M, Muchemwa FC, et al. Adiponectin expression is decreased in the involved skin and sera of diffuse cutaneous scleroderma patients. Exp Dermatol 2011; 20: 764-6.

42. Masui Y, Asano Y, Takahashi T, et al. Clinical significance of monitoring serum adiponectin levels during intravenous pulse cyclophosphamide therapy in interstitial lung disease associated with systemic sclerosis. Mod Rheumatol 2013; 23: 323-9.

43. Clements $P$, Lachenbruch P, Seibold J, et al. Inter and intraobserver variability of total skin thickness score (modified Rodnan TSS) in systemic sclerosis. J Rheumatol 1995; 22: 1281-5.

44. LeRoy EC, Black C, Fleischmajer R, et al. Scleroderma (systemic sclerosis): classification, subsets and pathogenesis. J Rheumatol 1988; 15: 202-5.

45. Valentini G, Della Rossa A, Bombardieri S, et al. European multicentre study to define disease activity criteria for systemic sclerosis. II. Identification of disease activity variables and development of preliminary activity indexes. Ann Rheum Dis 2001; 60: 592-8.

46. Cutolo M, Pizzorni C, Sulli A. Nailfold video-capillaroscopy in systemic sclerosis. Z Rheumatol 2004; 63: 457-62.

47. Voilliot D, Magne J, Dulgheru R, et al. Determinants of exercise-induced pulmonary arterial hypertension in systemic sclerosis. Int J Cardiol 2014; 173: 373-9.

48. Yalçindağ FN, Kisa U, Batioğlu F, et al. Serum leptin levels in patients with ocular and nonocular Behçet's disease. Mediators Inflamm 2007; 2007: 31986.

49. Garcia-Gonzalez A, Gonzalez-Lopez L, Valera-Gonzalez IC, et al. Serum leptin levels in women with systemic lupus erythematosus. Rheumatol Int 2002; 22: 138-41.

50. Wisłowska M, Rok M, Stępień K, Kukło-Kowalska A. Serum leptin in systemic lupus erythematosus. Rheumatol Int 2008; 28: 467-73

51. Mohammed SF, Abdalla MA, Ismaeil WM, Sheta MM. Serum leptin in systemic lupus erythematosus patients: its correlation with disease activity and some disease parameters. Egypt Rheumatol 2018; 40: 23-7.

52. Barbosa VdeS, Francescantônio PL, Da Silva NA. Leptin and adiponectin in patients with systemic lupus erythematosus: 
clinical and laboratory correlations. Rev Bras Reumatol 2015; 55: 140-5.

53. Wisłowska M, Rok M, Jaszczyk B, et al. Serum leptin in rheumatoid arthritis. Rheumatol Int 2007; 27: 947-54.

54. Evereklioglu C, Inalöz HS, Kirtak N, et al. Serum leptin concentration is increased in patients with Behçet's syndrome and is correlated with disease activity. Br J Dermatol 2002; 147: 331-6.

55. Fantuzzi G. Adiponectin and inflammation: consensus and controversy. J Allergy Clin Immunol 2008; 121: 326-30.

56. Šenolt L, Pavelka K, Housa D, Haluzík M. Increased adiponectin is negatively linked to the local inflammatory process in patients with rheumatoid arthritis. Cytokine 2006; 35: 247-52.

57. Medoff BD, Okamoto Y, Leyton P, et al. Adiponectin deficiency increases allergic airway inflammation and pulmonary vascular remodeling. Am J Respir Cell Mol Biol 2009; 41: 397-406.

58. De Sanctis JB, Zabaleta M, Bianco NE, et al. Serum adipokine levels in patients with systemic lupus erythematosus. Autoimmunity 2009; 42: 272-4.

59. Rho YH, Solus J, Sokka T, et al. Adipocytokines are associated with radiographic joint damage in rheumatoid arthritis. Arthritis Rheum 2009; 60: 1906-14.

60.Toussirot EE, Gaugler B, Bouhaddi M, et al. Elevated adiponectin serum levels in women with systemic autoimmune diseases. Mediators Inflamm 2010; 2010: 938408.

61. Chizzolini C, Brembilla NC, Montanari E, Truchetet ME. Fibrosis and immune dysregulation in systemic sclerosis. Autoimmun Rev 2011; 10: 276-81.

62. Dagog-Jack S, Fanelli C, Paramore D, et al. Plasma leptin and insulin relationships in obese and nonobese humans. Diabetes 1996; 45: 695-8.

63. Toussirot E, Streit G, Nguyen NU, et al. Adipose tissue, serum adipokines, and ghrelin in patients with ankylosing spondylitis. Metabolism 2007; 56: 1383-9.

64.Sari I, Demir T, Kozaci LD, et al. Body composition, insulin, and leptin levels in patients with ankylosing spondylitis. Clin Rheumatol 2007; 26: 1427-32.

65. Gómez-Bańuelos E, Navarro-Hernández RE, Corona-Meraz F, et al. Serum leptin and serum leptin/serum leptin receptor ratio imbalance in obese rheumatoid arthritis patients positive for anti-cyclic citrullinated peptide antibodies. Arthritis Res Ther 2015; 17: 335.

66. Marangoni RG, Masui Y, Fang F, et al. Adiponectin is an endogenous anti-fibrotic mediator and therapeutic target. Sci Rep 2017; 7: 4397.

67. Miller M, Cho JY, Pham A, et al. Adiponectin and functional adiponectin receptor 1 are expressed by airway epithelial cells in chronic obstructive pulmonary disease. J Immunol 2009; 182: 684-91.

68.Dong Z, Su L, Esnaili S, et al. Adiponectin attenuates liver fibrosis by inducing nitric oxide production of hepatic stellate cells. J Mol Med (Berl) 2015; 93: 1327-39.

69. Yao R, Cao Y, He YR, et al. Adiponectin attenuates lung fibroblasts activation and pulmonary fibrosis induced by paraquat. PLoS One 2015; 10: e0125169.

70. Fang F, Liu L, Yang Y, et al. The adipokine adiponectin has potent anti-fibrotic effects mediated via adenosine monophosphate-activated protein kinase: novel target for fibrosis therapy. Arthritis Res Ther 2012; 14: R229.

71. Saxena NK, Anania FA. Adipocytokines and hepatic fibrosis. Trends Endocrinol Metab 2015; 26: 153-61.
72. Handy JA, Saxena NK, Fu P, et al. Adiponectin activation of AMPK disrupts leptin-mediated hepatic fibrosis via suppressors of cytokine signaling (SOCS-3). J Cell Biochem 2010; 110: 1195-207.

73. Jain M, Budinger GR, Lo A, et al. Leptin promotes fibroproliferative acute respiratory distress syndrome by inhibiting peroxisome proliferator-activated receptor-gamma. Am J Respir Crit Care Med 2011; 183: 1490-8.

74. Vivoli E, Di Maira G, Marra F. Liver fibrosis and leptin. Curr Pathobiol Rep 2016; 4: 69-76.

75. Targońska-Stępniak B, Majdan M, Dryglewska M. Leptin serum levels in rheumatoid arthritis patients: relation to disease duration and activity. Rheumatol Int 2006; 99: 585-91.

76. Lee SW, Spark MC, Park YB, Lee SK. Measurement of the serum leptin level could assist disease activity monitoring in rheumatoid arthritis. Rheumatol Int 2007; 27: 537-40.

77. Xibillé-Friedmann DX, Ortiz-Panozo E, Bustos Rivera-Bahena C, et al. Leptin and adiponectin as predictors of disease activity in rheumatoid arthritis. Clin Exp Rheumatol 2009; 27: 222-8.

78. Park MC, Lee SW, Choi ST, et al. Serum leptin levels correlate with interleukin- 6 levels and disease activity in patients with ankylosing spondylitis. Scand J Rheumatol 2007; 36: 101-6.

79. Gabay C, Dreyer M, Pellegribelli N, et al. Leptin directly induces the secretion of interleukin 1 receptor antagonist in human monocytes. J Clin Endocrinol Metab 2001; 86: 783-91.

80.Cakir B, Bozkurt A, Ercan F, Yeğen BC. The anti-inflammatory effect of leptin on experimental colitis: involvement of endogenous glucocorticoids. Peptides 2004; 25: 95-104.

81. Popa C, Netea MG, Radstake TR, et al. Markers of inflammation are negatively correlated with serum leptin in rheumatoid arthritis. Ann Rheum Dis 2005; 64: 1195-8.

82. Sierra-Hinigmann MR, Nath AK, Murakami C, et al. Biological action of leptin as an angiogenic factor. Science 1998; 281: 1683-6.

83. Park HY, Kwon HM, Lim HJ, et al. Potential role of leptin in angiogenesis: leptin induces endothelial cell proliferation and expression of matrix metalloproteinases in vivo and in vitro. Exp Mol Med 2001; 33: 95-102.

84. Yang WH, Chen JC, Hsu KH, et al. Leptin increases VEGF expression and enhances angiogenesis in human chondrosarcoma cells. Biochim Biophys Acta 2014; 1840: 3483-93.

85. Del Turco S, Navarra T, Gastadelli A, Basta G. Protective role of adiponectin on endothelial dysfunction induced by AGEs: a clinical and experimental approach. Microvasc Res 2011; 82: 73-6.

86.Zubair M, Malik A, Ahmad J. Plasma adiponectin, IL-6, hsCRP, and TNF-alpha levels in subject with diabetic foot and their correlation with clinical variables in a North Indian tertiary care hospital. Indian J Endocrinol Metab 2012; 16: 769-76. 